\title{
Investigation of Psychometric Properties of Misophonia Questionnaire
}

\author{
Mahnaz Mehrabizadeh Honarmand, Khadijeh Roushani*
}

Department of Psychology, Faculty of Education and Psychology, Shahid Chamran University of Ahvaz, Ahvaz, Iran

\section{A BSTRACT}

Introduction: Misophonia is a condition in which individuals react negatively to sounds that occur in particular situations, but they tolerate other sounds that are frequently much louder. The present research was conducted to investigate of psychometric properties of Misophonia Questionnaire in college students in order to provide a tool for evaluation in the research process and used by researchers and therapists. Materials and Methods: The sample of this study was 319 undergraduate students (176 females and 143 male) who were selected from students of Payame Noor University of Ahvaz by multi-stage sampling method. The instruments of this study consisted of Misophonia Questionnaire of Wu, Lewin, Murphy \& Storch, Yale-Brown Obsessive Compulsive Scale, Beck Anxiety Inventory (BAI-II) and Beck Depression Inventory (BDI-II). Results: The internal consis tency with Cronbach's alpha and correlation of each questionnaire's items with total score of scale showed acceptable reliability. In addition, confirmatory factor analysis showed that two-factor structure of this scale was appropriate. Furthermore, the results of convergent validity indicated that Misophonia Questionnaire has a significant positive relationship with Yale-Brown Obsessive Compulsive Scale, Beck Anxiety Inventory (BAI-II) and Beck Depression Inventory (BDI-II). Conclusion: The results showed that Misophonia Questionnaire is a valid and reliable scale for measuring misophonia in Iranian student.

Key words:

1. Anxiety

2. Depression

3. Surveys and Questionnaires

*Corresponding Author: Khadijeh Roushani

E-mail: kh_roushani@yahoo.com 


\title{
بررسى ويزگَى هاى روانسنجى يرسشنامهُ صدا بيزارى
}

\author{
مهناز مهر ابى زاده هنرمند، خديجه روشنى" \\ كروه روانشناسى، دانشكده علوم تربيتى و روانشناسى، دانشكاه شهيد جمران اهواز، اهواز، ايران
}

\section{اطلاعات مقاله:}

تاريخ يذيرش: وج بهمن Vqr

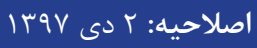

تاريخ دريافت: آ مهر Vqr|

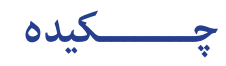

مقدمه: صدا بيزارى حالتى است كه در آن افراد نسبت به صداهايى كه در موقعيتهاى بهخصوصى رخ

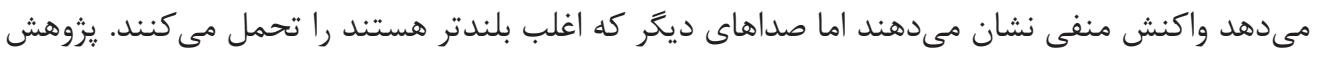

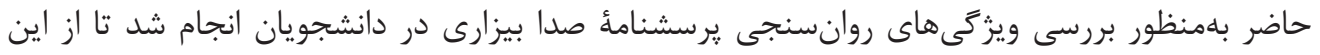

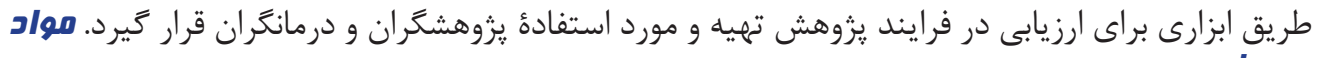

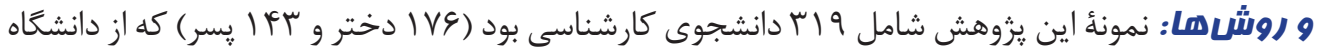

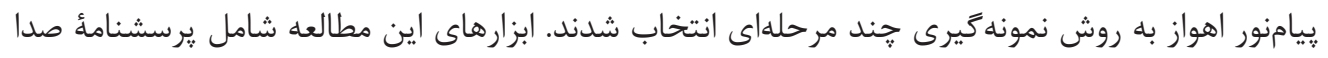

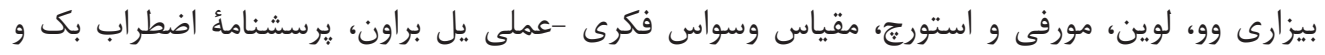

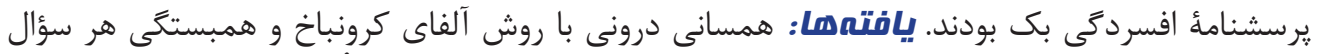

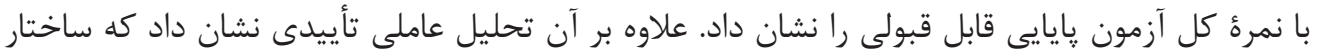

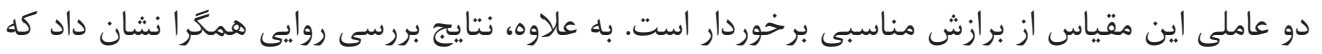

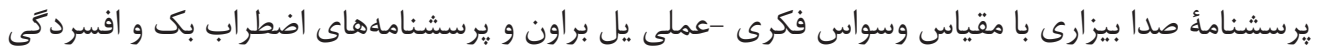

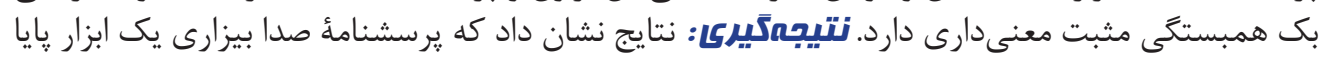
و معتبر براى سنجش صدا بيزارى دانشجويان ايرانى مئى داشد.

ب.

كليد وازهها:

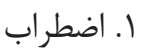

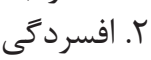

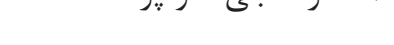

" نويسنده مسئول: خديجه روشنى

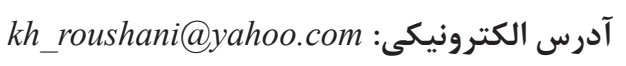




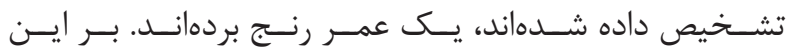

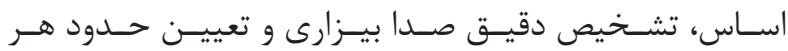

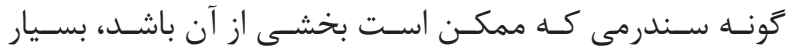

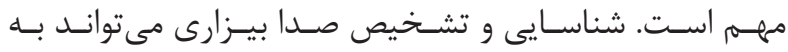

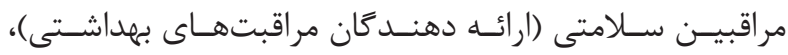

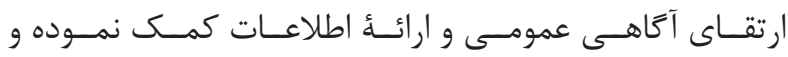

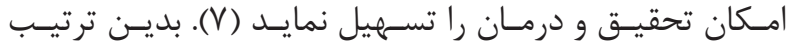

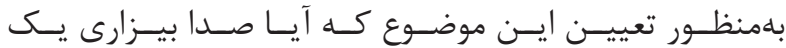

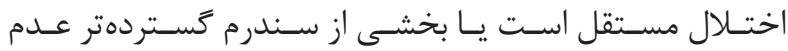

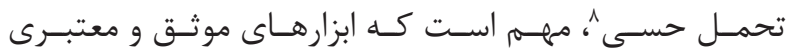

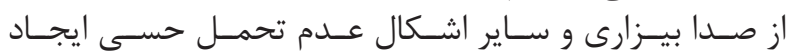

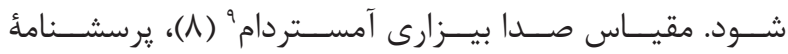

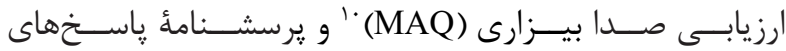

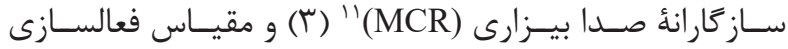

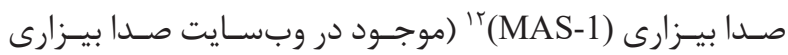

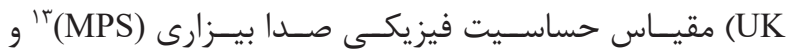

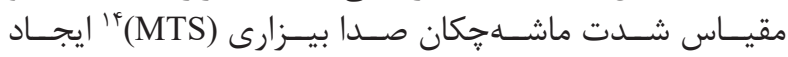

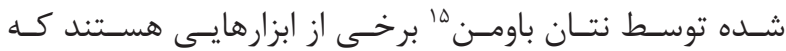

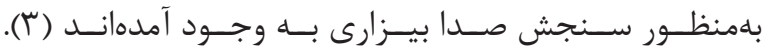

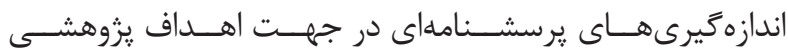

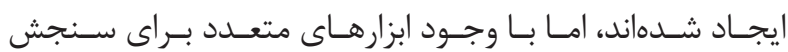

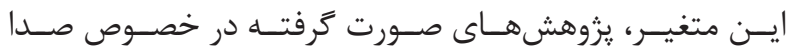

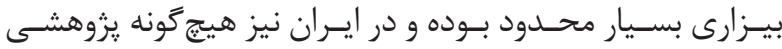

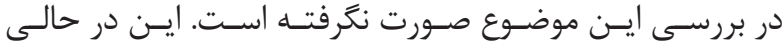

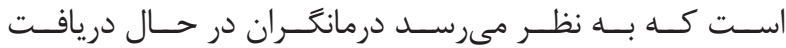

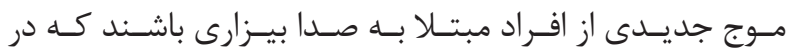

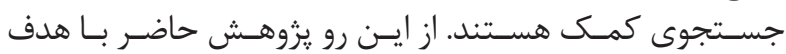

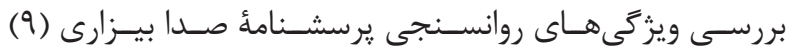

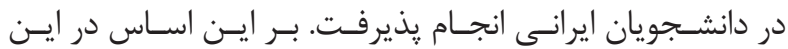

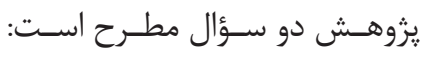

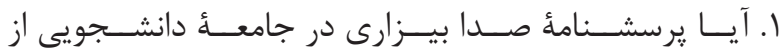

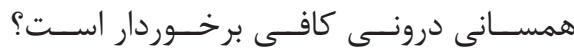

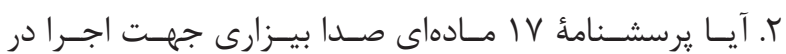

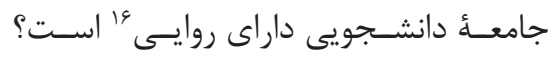

مواد و روشها

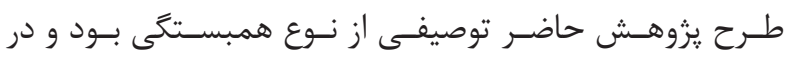

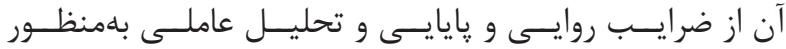

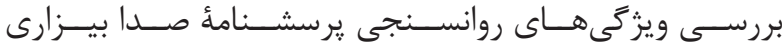

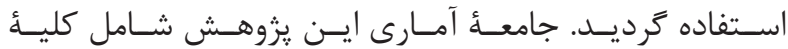

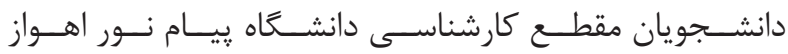

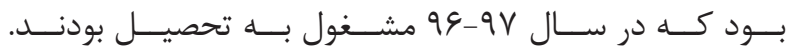

${ }^{1}$ Misophonia

${ }^{2}$ Jastreboff

${ }^{3}$ Dozier

${ }^{4}$ Tigger

${ }^{5}$ Lips smacking

${ }^{6}$ Selective sound sensitivity syndrome

${ }^{7}$ Soft sound sensitivity syndrome

${ }^{8}$ Sensory intolerance syndrome

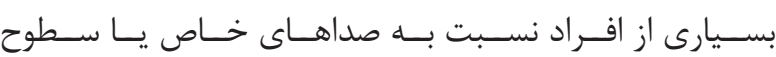

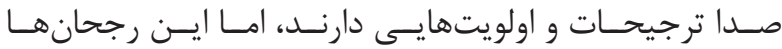

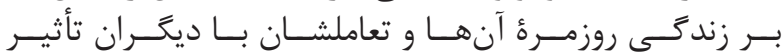

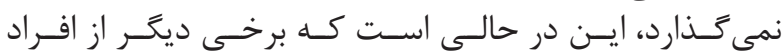

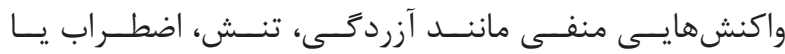

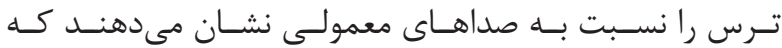

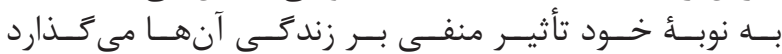

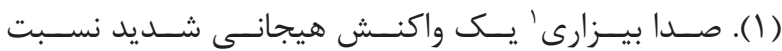

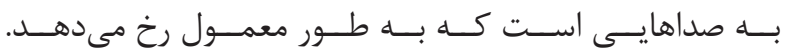

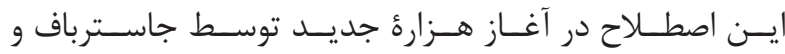

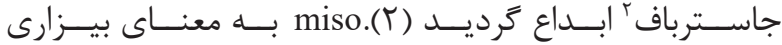

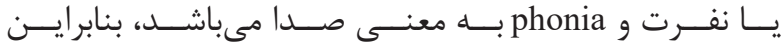
misophonia

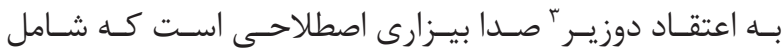

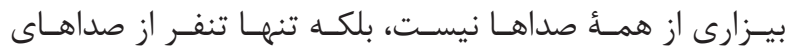

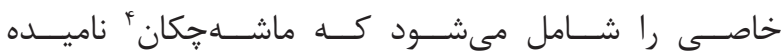

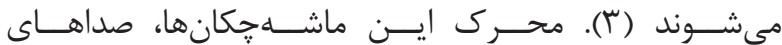

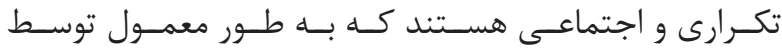

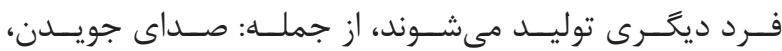

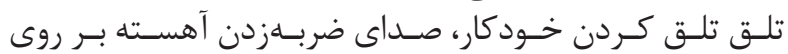

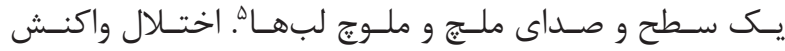

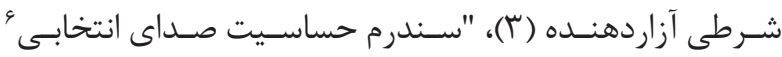

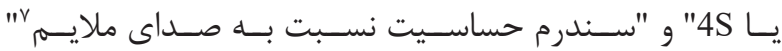

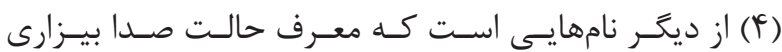

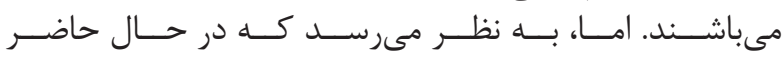

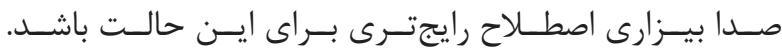

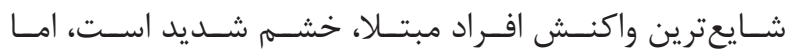

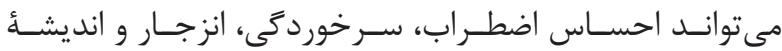

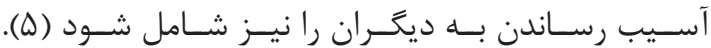

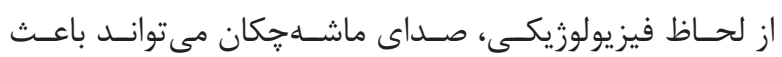

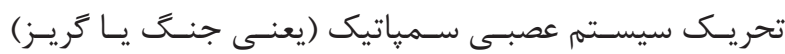

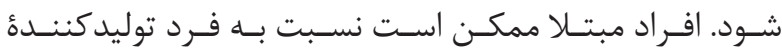

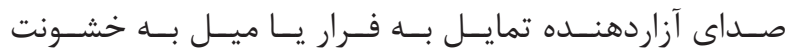

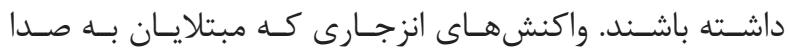

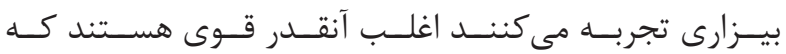

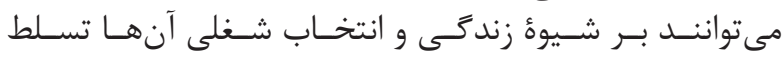

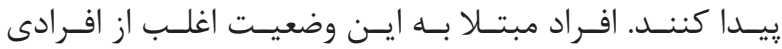

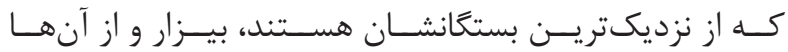

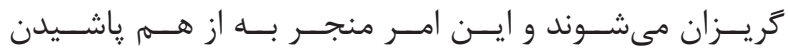

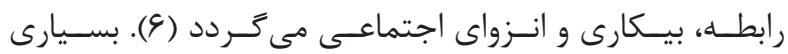

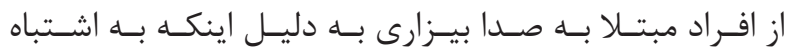

\footnotetext{
${ }^{9}$ Amsterdam misophonia scale

${ }^{10}$ Misophonia assessment questionnaire

${ }^{11}$ Misophonia coping responses

${ }^{12}$ Misophonia activation scale

${ }^{13}$ Misophonia physical sensation scale

${ }^{14}$ Misophonia trigger severity scale

${ }^{15}$ Natan bauman

${ }^{16}$ Validity
} 


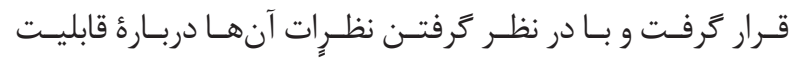

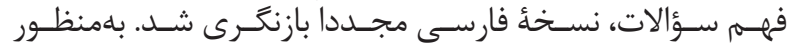

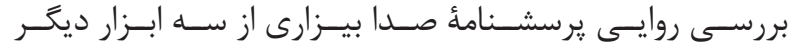

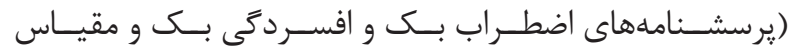

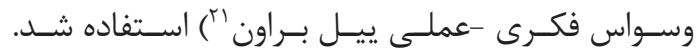

\section{يرسشنامهُ اضطراب بك نسخهُ دوم}

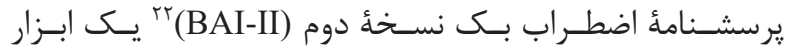

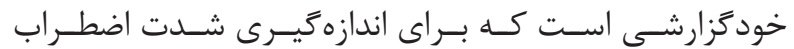

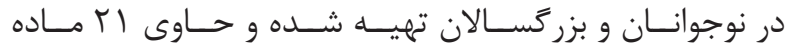

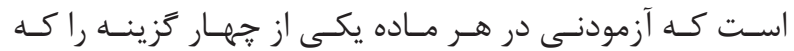

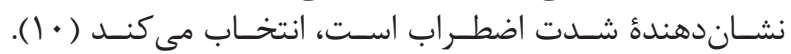

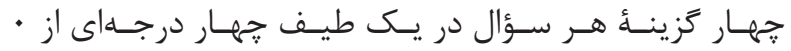

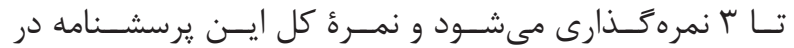

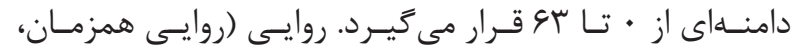

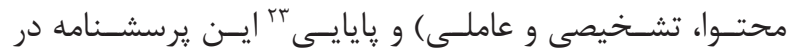

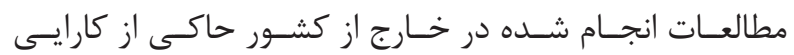

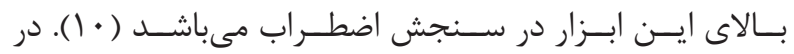

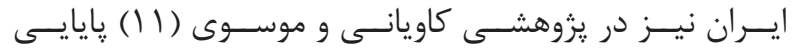

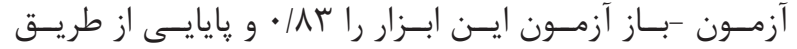

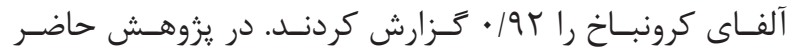

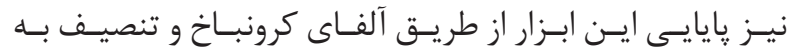

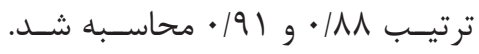

يرسشنامة افسردَى بك نسخة دوم

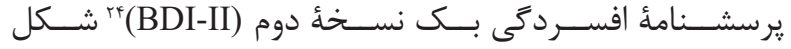

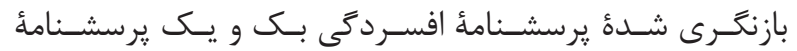

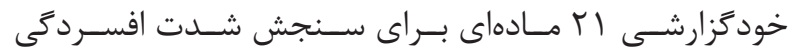

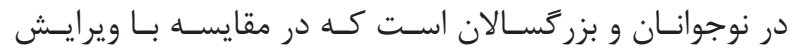

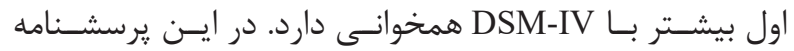

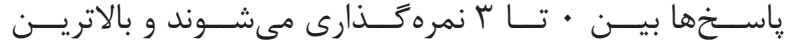

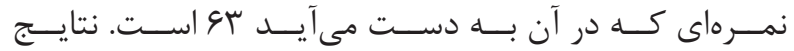

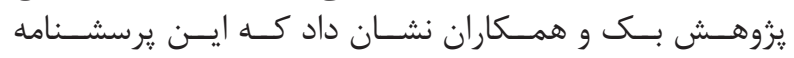

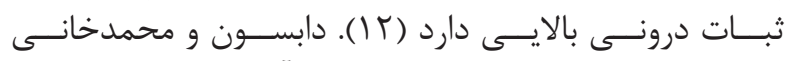

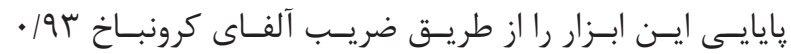

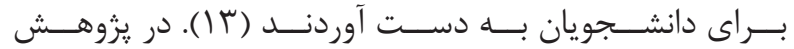

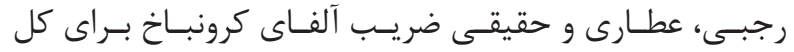

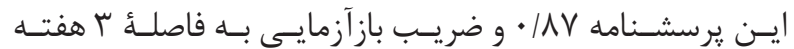

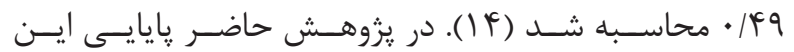

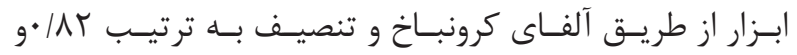

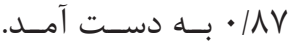

\section{مقياس وسواس فكرى -عملى يل براون}

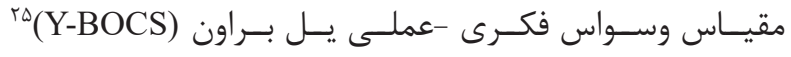

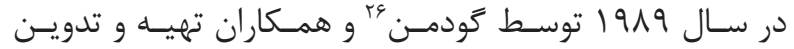

\section{${ }^{17}$ Krejcie}

${ }^{18}$ Morgan

${ }^{19}$ Misophonia questionnaire

${ }^{20}$ The national institute of mental health-global obsessivecompulsive scale

${ }^{21}$ Yale-brown obsessive compulsive scale

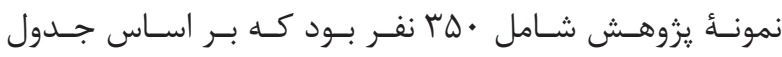

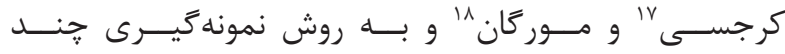

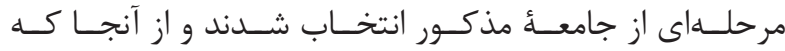

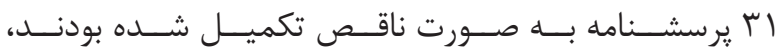

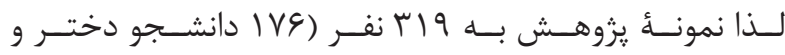

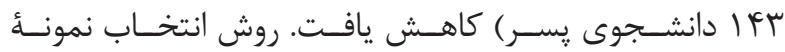

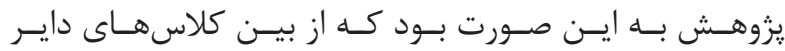

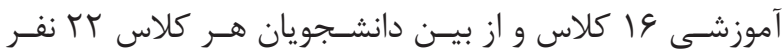

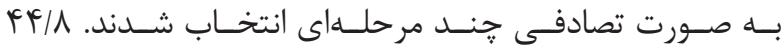

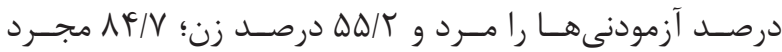

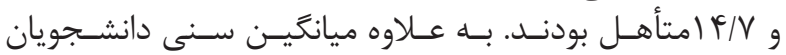

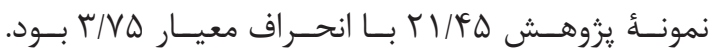

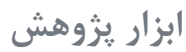

\section{يرسشنامة صدا بيزارى}

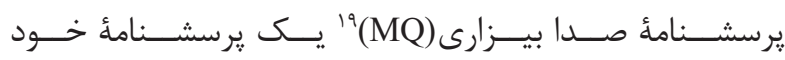

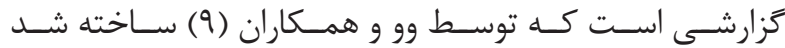

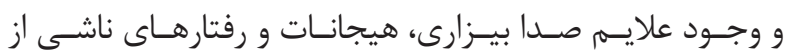

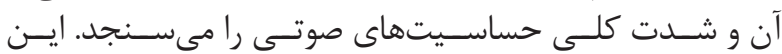

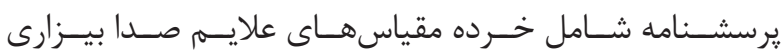

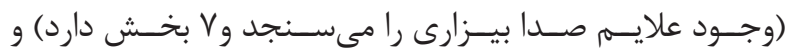

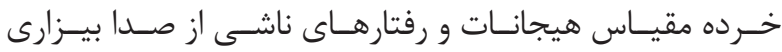

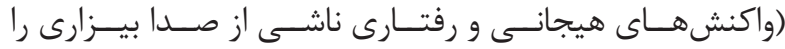

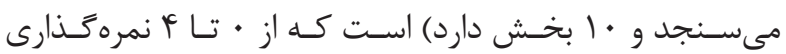

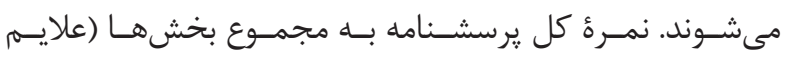

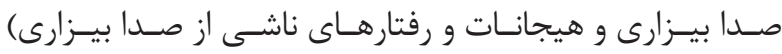

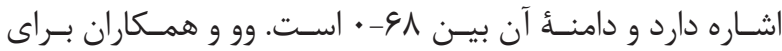

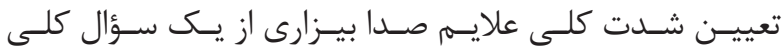

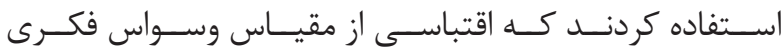

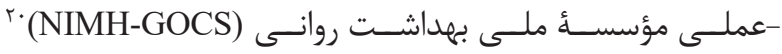

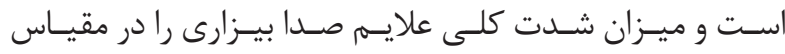

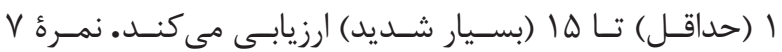

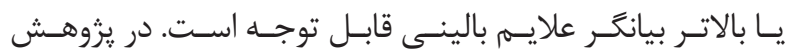

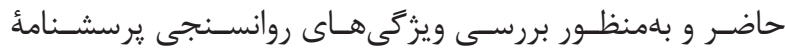

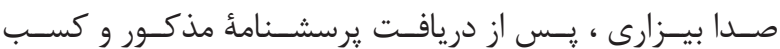

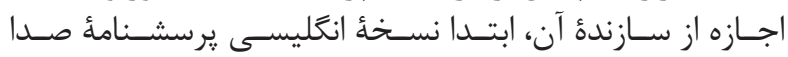

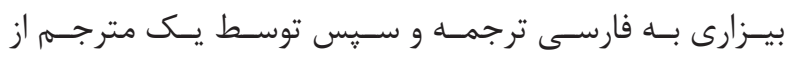

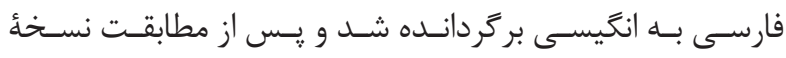

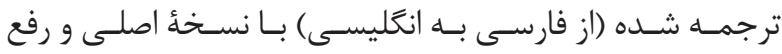

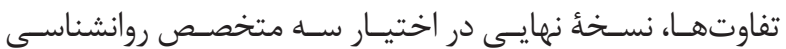

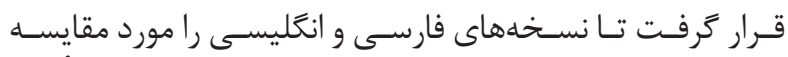

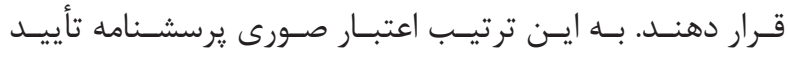

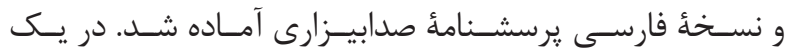

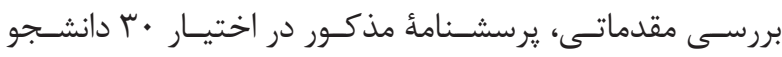

${ }^{22}$ Beck anxiety inventory

${ }^{23}$ Reliability

${ }^{24}$ Beck depression inventory

${ }^{25}$ Yale-brown obsessive compulsive scale

${ }^{26}$ Goodman 
صــا بيـزارى را نشــان مى دهــد.

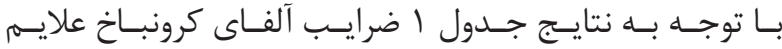

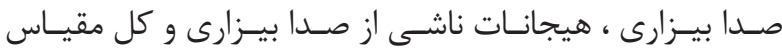

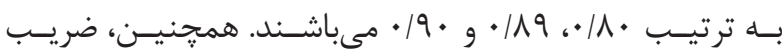

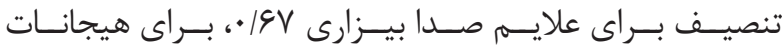

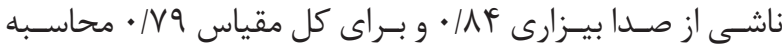

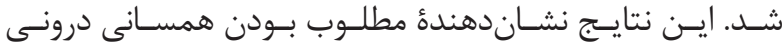

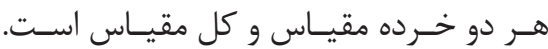

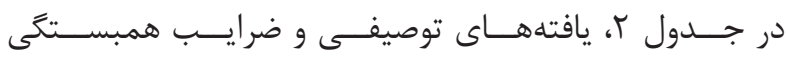

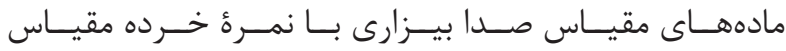

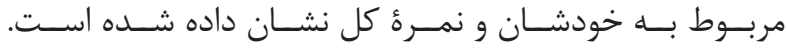

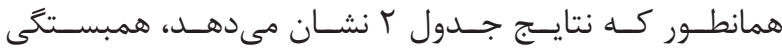

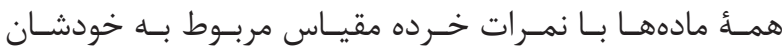

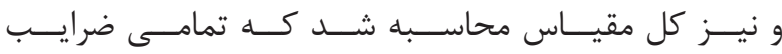

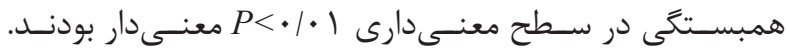

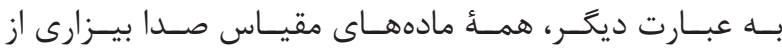

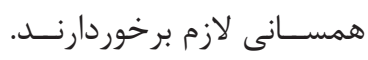

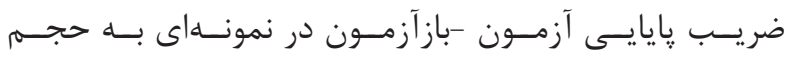

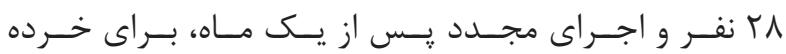

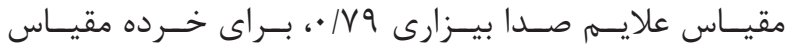

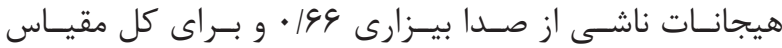

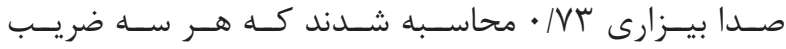

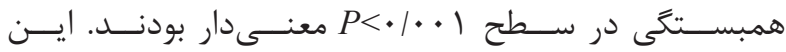

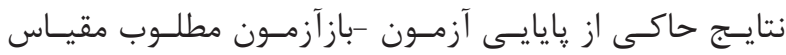

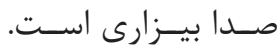

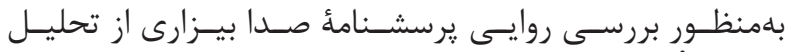

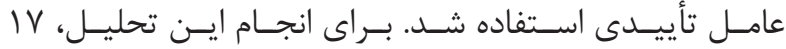

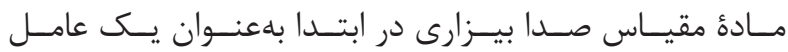

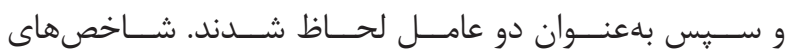

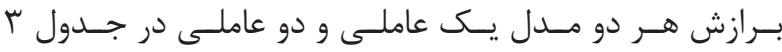

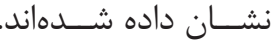

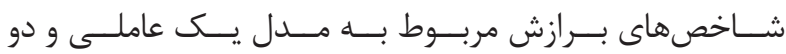

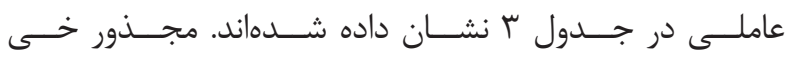

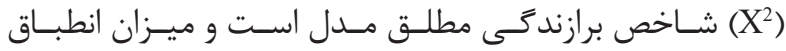

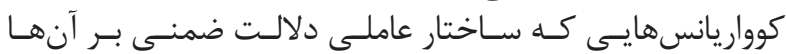

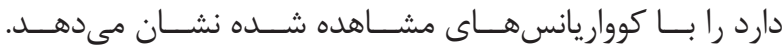

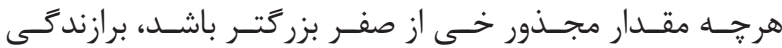

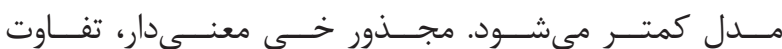

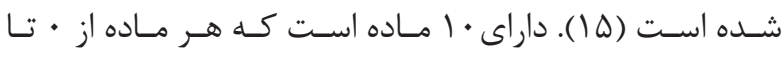

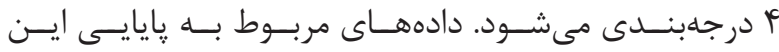

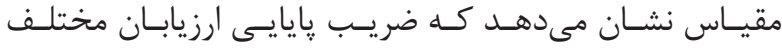

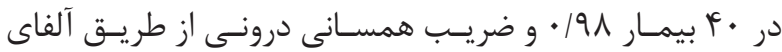

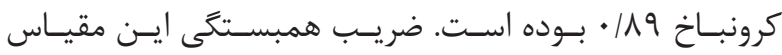

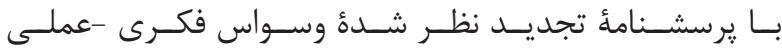

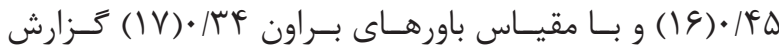

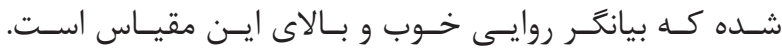

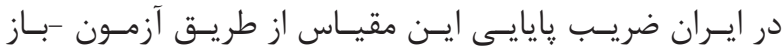

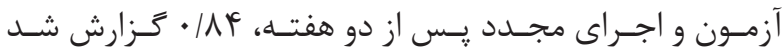

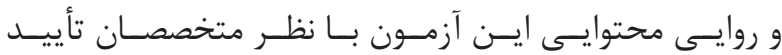

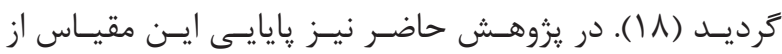

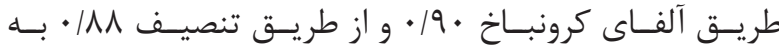
دسـت آمـد.

تجزيه و تحليل دادهها

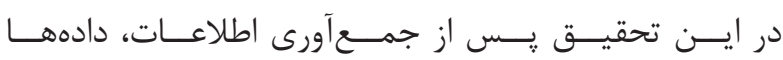

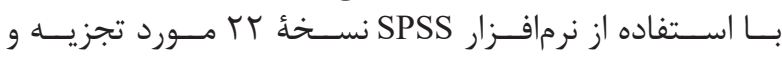

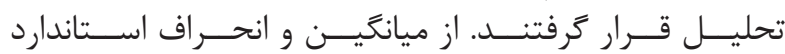

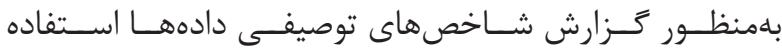

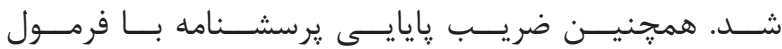

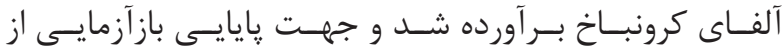

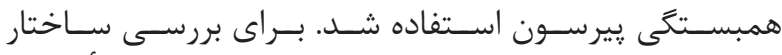

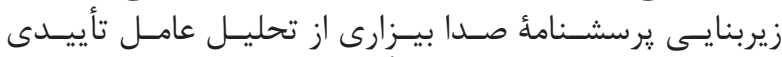

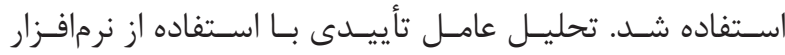

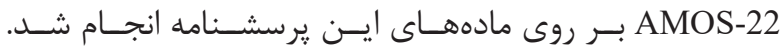

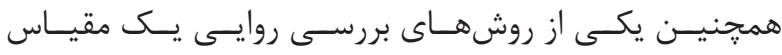

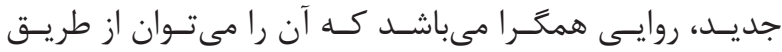

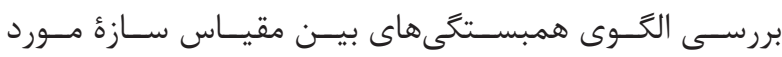

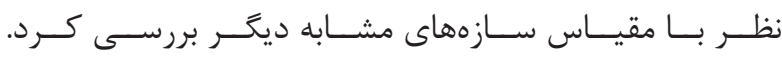

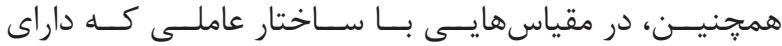

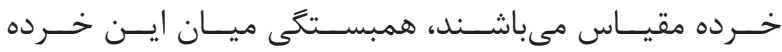

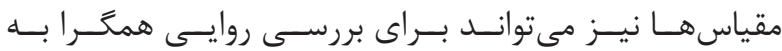

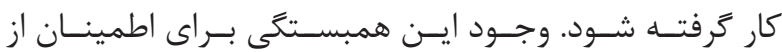

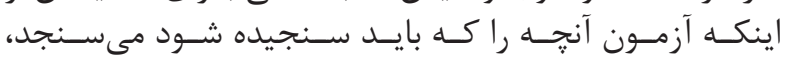
ضـرورى اسـت أنست

بافتهها

جــدول ا، يافتههــاى توصيفـى و ضرايسب آلفــاى كرونبــاخ و

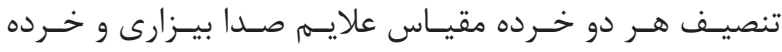

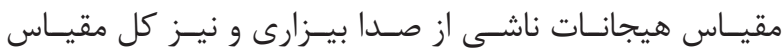

جدول ا- يافتهاى توصيفى و ضرايب يايايى خرده مقياسها و كل مقياس صدا بيزارى (n=r|9).

\begin{tabular}{|c|c|c|c|}
\hline ضريب تنصيف & ضريب آلفاى كرونباخ & انحراف معيار 土ـميانكين & 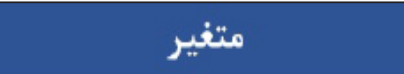 \\
\hline $.19 V$ & $\cdot / \Lambda$. & $1.19 V \pm 0 / 9 r$ & علايم صدا بيزارى \\
\hline$\cdot \mid A F$ & .119 & $|r / \Delta| \pm N / r q$ & هيجانات ناشى از صدا بيزارى \\
\hline.$/ 189$ & .19 & $r r / 19 \pm 1 r / 99$ & كل مقياس \\
\hline
\end{tabular}




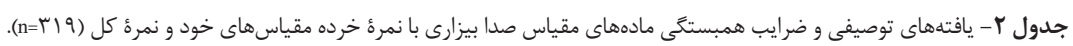

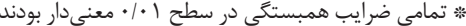

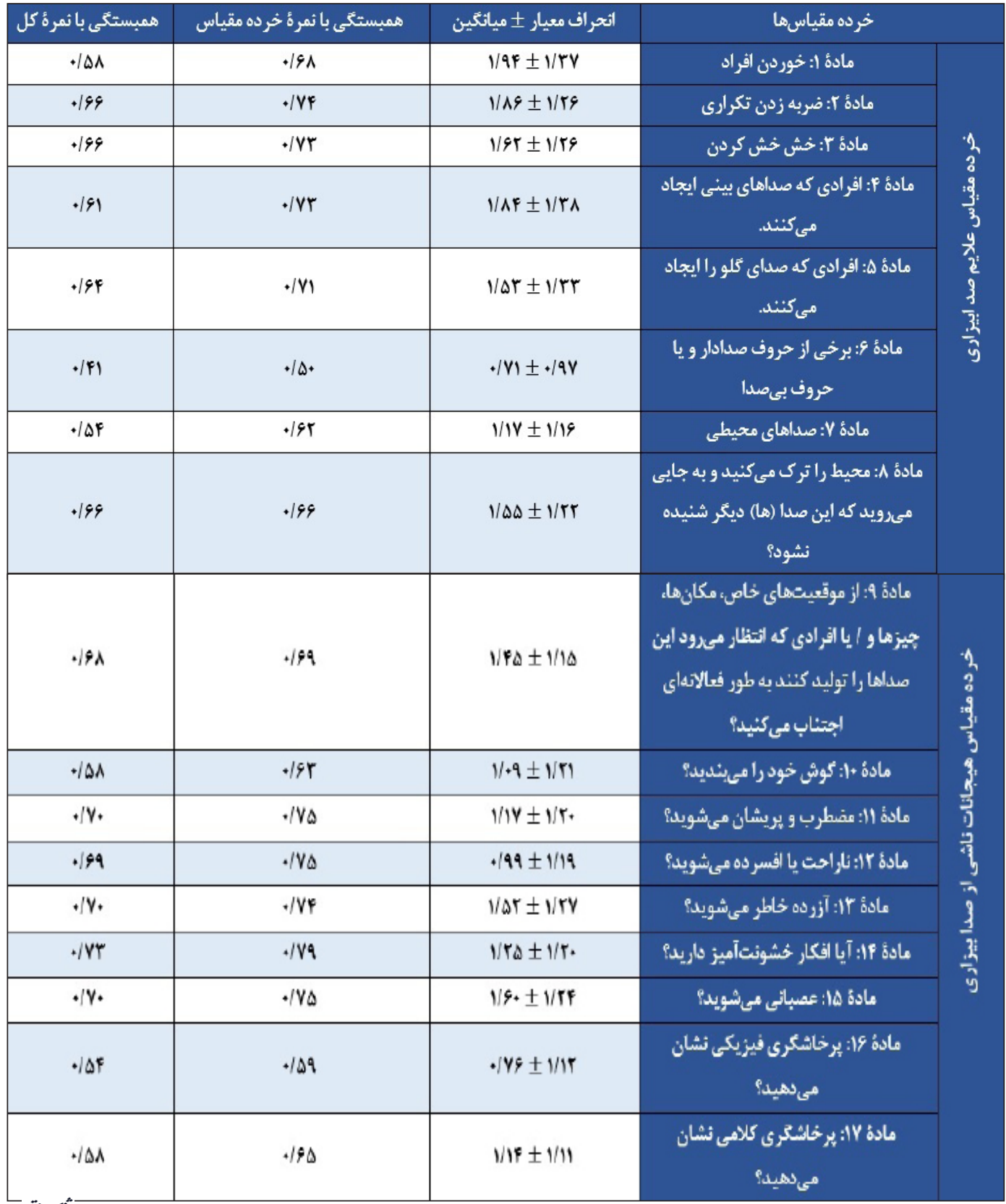

جدول بـ - شاخصهاى برازش ساختار يك عاملى و دو عاملى مقياس صدا بيزارى.

\begin{tabular}{|c|c|c|c|c|c|c|c|c|c|c|c|}
\hline RMSEA & NFI & CFI & TLI & IFI & GFI & AGFI & $\chi^{\gamma} / \mathbf{D F}$ & $\mathbf{P}$ & DF & $\chi^{x}$ & ساختار \\
\hline$\cdot \mid \cdot v_{1}$ & .11999 &.$/ 917$ & - $/ 194 \Delta$ &.$/ 91 \Delta$ & ./A9r & - IA $\Delta r$ & $r / 919$ & $\cdot / \cdot+1$ & 114 & $r q r / \cdot r q$ & يك عاملى \\
\hline. $\mid \cdot \Delta 1$ &.$/ 911$ & $\cdot / 9 \Delta \wedge$ & ./AFV &.$/ 9 \Delta A$ & $. / 9 .$. & - IADT & זוA/ & $\cdot / \cdot .1$ & 1.9 & $19 V / 95 V$ & دو عاملى \\
\hline
\end{tabular}

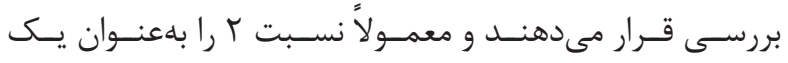

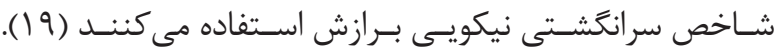

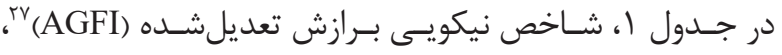

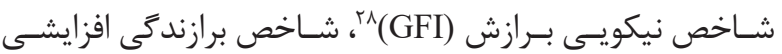

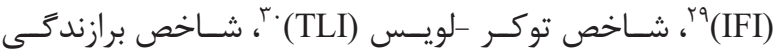

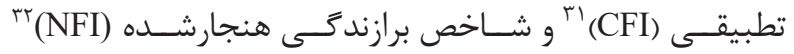

${ }^{27}$ Adjusted goodness of fit index

${ }^{28}$ Goodness of fit index

${ }^{29}$ Incremental fit index

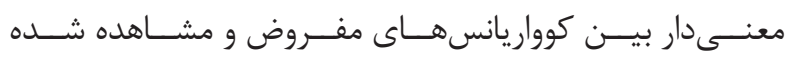

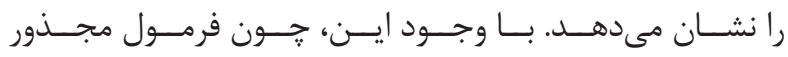

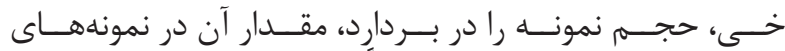

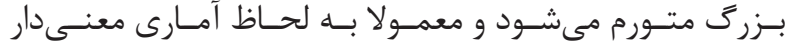

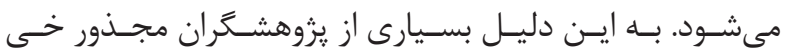

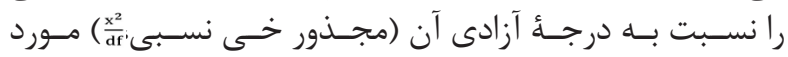

${ }^{30}$ Tucker-lewis index

${ }^{31}$ Comparative fit index

${ }^{32}$ Normed fit index 


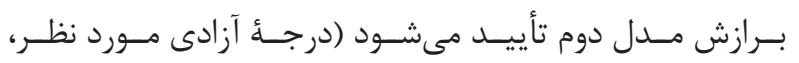

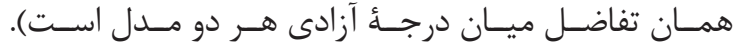

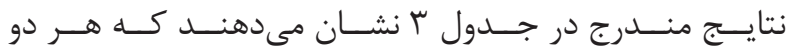

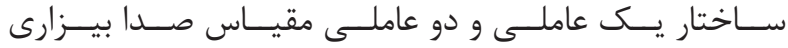

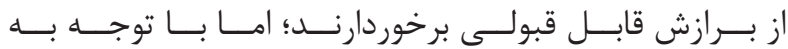

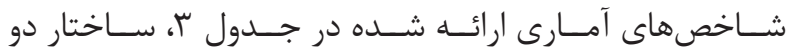

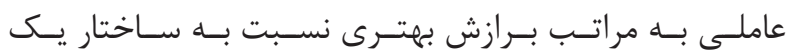

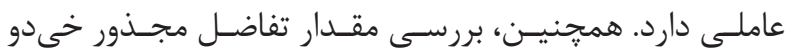

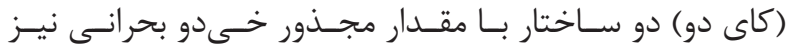

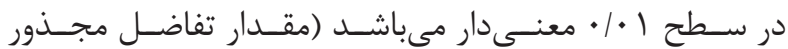

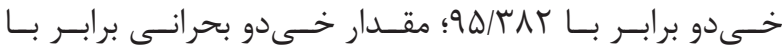

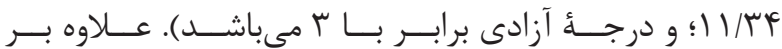

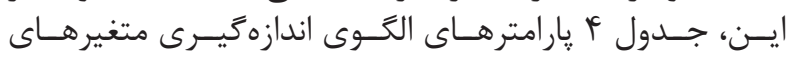

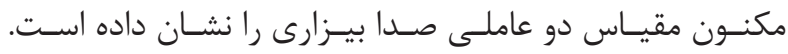

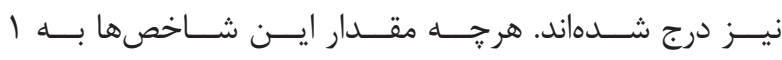

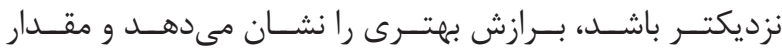

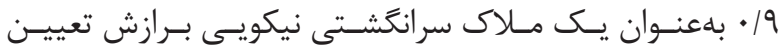

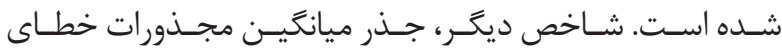

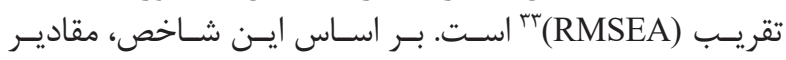

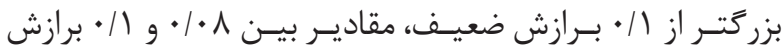

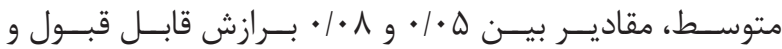

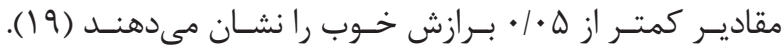

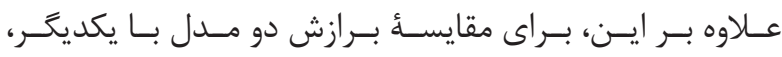

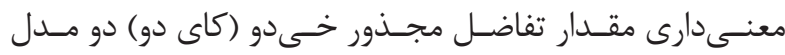

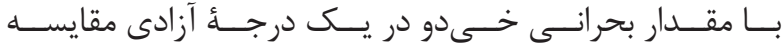

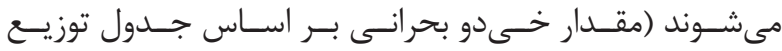

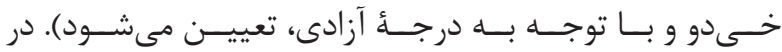

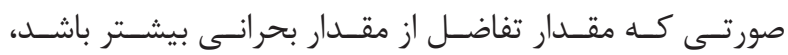

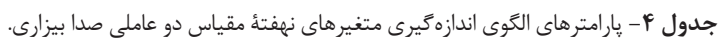

\begin{tabular}{|c|c|c|c|c|c|c|}
\hline معنى سمارى & نسبت بحرائى & خطاى معيار & برآورد هارامتر & يارامتر الستاندارد & 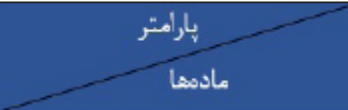 & لملما \\
\hline & & - & $1 / \cdots$ & +109 & مادة أ خوردن القران & \multirow{7}{*}{$\frac{3}{3}$} \\
\hline$+\rho+1$ & Q/TE & $\cdot / 1$ & $y \cdot 4$ & 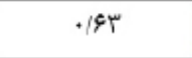 & مادة ז: فربه زني تكرارى & \\
\hline 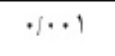 & NTF & $\cdot / \pi$ & $\mathrm{V} \cdot \mathrm{V}$ & .199 & مادة با: خشى خش كردن & \\
\hline$\cdot / \cdots 1$ & NF. & $\cdot / / F$ & $M / M$ & .199 & ماهة f: الفر الى كه مشاهاى بينى ايجاه & \\
\hline$\cdot \gamma \cdot 1$ & $N F D$ & $\cdot / 14$ & Whe & $\cdot 19 \mathrm{~V}$ & عادة ه: الثرادى كه صداى كلو را ايجاد & \\
\hline$\cdot \% \cdots$ & 81. &.$/ \cdot 9$ & $\cdot \mid \Delta r$ &.$|4|$ & مادة و: برخى ازز حروف صدادار و يا & \\
\hline$+1+1$ & V/ef & $\cdot / 1$ & ז & $\cdot / \Delta \Delta$ & مادة V: صداماي محمبطى & \\
\hline - & - & - & $\| \cdots$ & $\cdot / \Delta q$ & 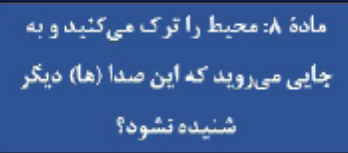 & \multirow{10}{*}{$\begin{array}{c}g_{1} \\
\frac{5}{5} \\
\frac{3}{3} \\
\frac{3}{43} \\
\overline{3} \\
\frac{3}{3} \\
\frac{3}{3}\end{array}$} \\
\hline$\cdot * 1$ & $11 / 19$ & $\cdot / \cdot 9$ & $\mid / \cdots)$ & ז & 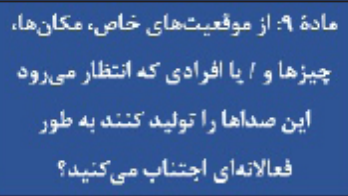 & \\
\hline$+\cdots+1$ & $N|*|$ &.$/ 14$ &.$/ 91$ & $\cdot / \Delta \Lambda$ & عادة +1: كوثى خوه راغينديد؟ & \\
\hline$\cdot \%+1$ & 9/V &.$/ 14$ & $1 / 11$ & $\cdot / Y$. & ماهة 11: عضطر ب و ير يشان مي شويده & \\
\hline$+1+\cdots$ & 9/AT & $\cdot / 14$ & $1 / 19$ & $\cdot|Y|$ & مادة كا: نار الحت يا القسر ده عي شويدا؟ & \\
\hline$+1+\cdots$ & $1 \cdot 1 \cdot r$ & ./ז & $1 / 4$ & $\cdot / \mathrm{VF}$ & ماد كا: آزر مه خاطر ميثويد؟ & \\
\hline$* \cdots 1$ & $1 \cdot / 4 r$ &.$/ 1 T$ & $1 / r$ & $\cdot / Y V$ & 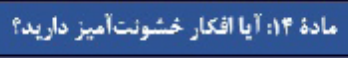 & \\
\hline$\cdot \% \cdot 1$ & $1 \cdot 1 \cdots$ & $\cdot / 1 T$ & $1 / 59$ & $\cdot / Y F$ & مادة ها: عصبانى مىشويد؟ & \\
\hline$\cdot \mu 1$ & V/av & $\cdot / 1$ & $\cdot|\lambda|$ & $\cdot[\Delta r$ & مادة 19: يرخاشكرى فيزيكى نشان & \\
\hline$\cdot \cdots 1$ & NTH & $\cdot / 1$ & $\cdot \mid M A$ & $\cdot / \Delta \varphi$ & مادة 1V: برخاشكرى كلامى نشان & \\
\hline
\end{tabular}

${ }^{33}$ Root mean square error of approximation 
بحث و نتيجه

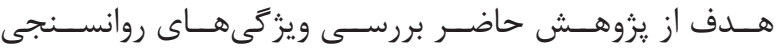

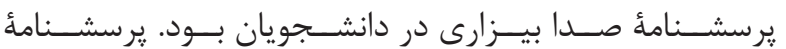

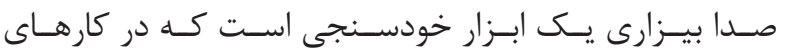

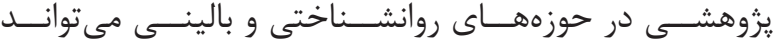

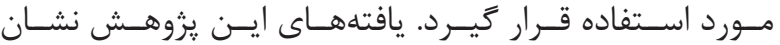

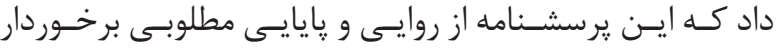

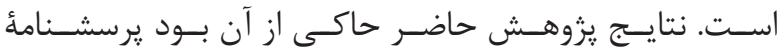

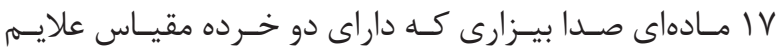

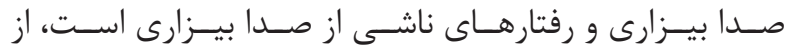

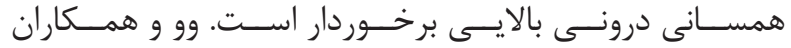

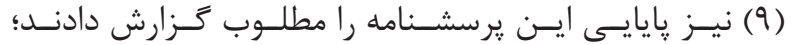

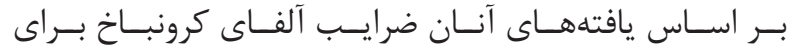

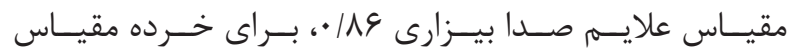

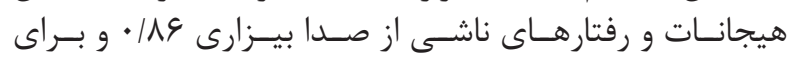

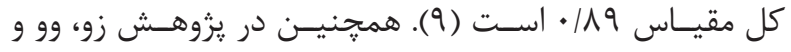

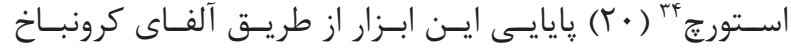

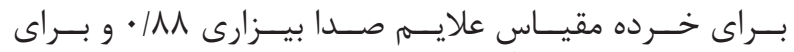

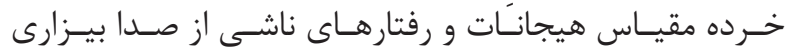

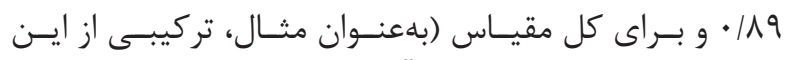

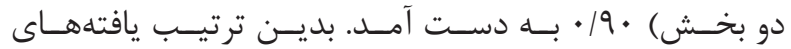

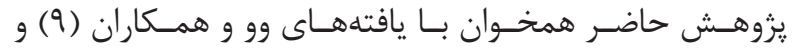

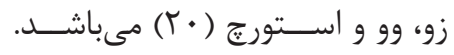

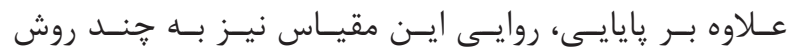

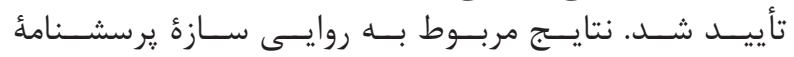

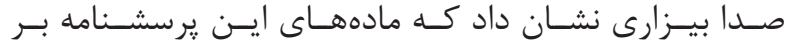

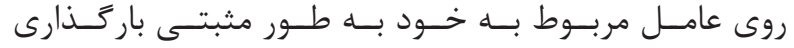

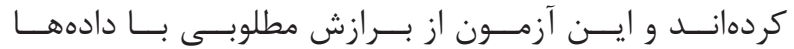

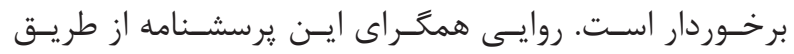

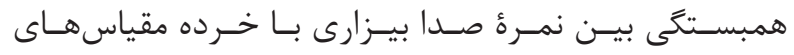

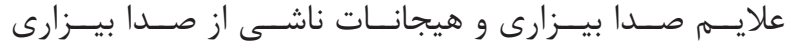

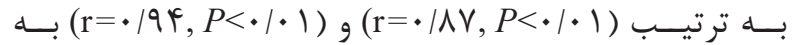

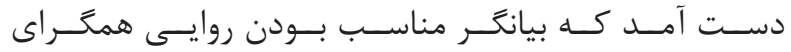

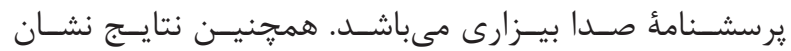

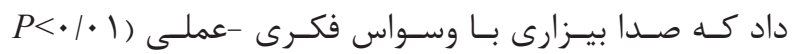

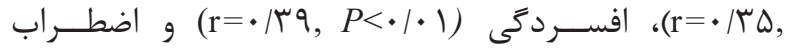

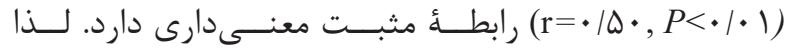

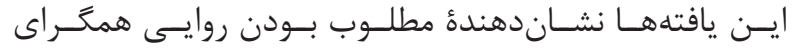

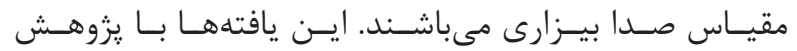

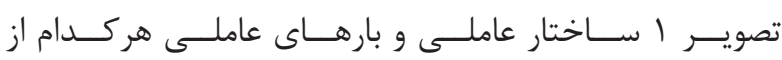

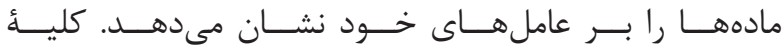

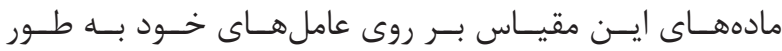

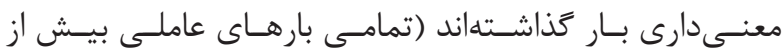

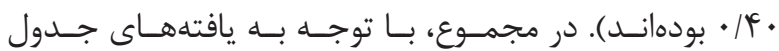

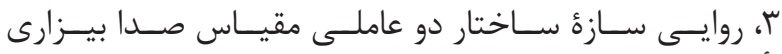

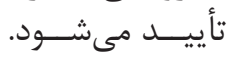

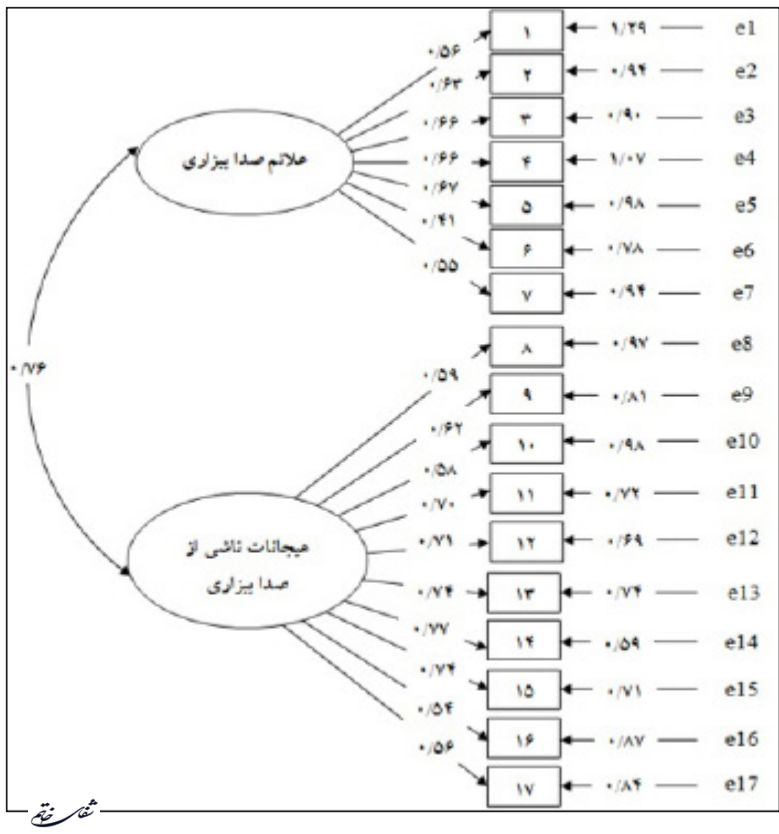

تصوير ا- ساختار عاملى و بارهاى عاملى هر يك از مادهها بر عاملهاى خود.

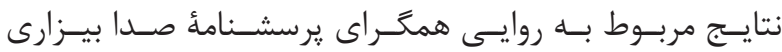

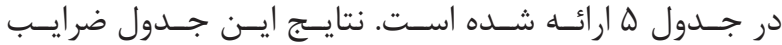

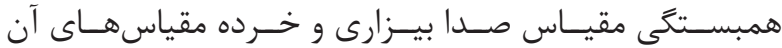

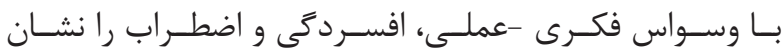

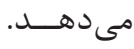

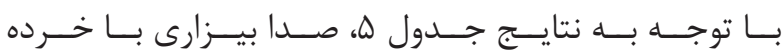

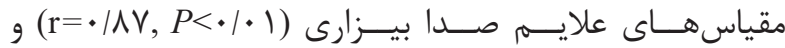

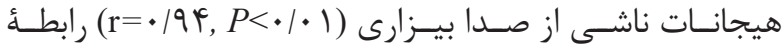

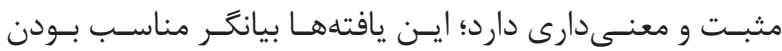

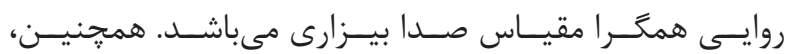

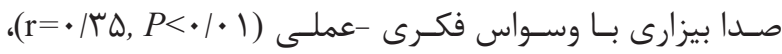

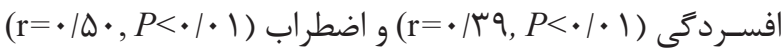

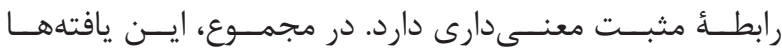

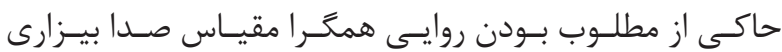
مىباشـــند.

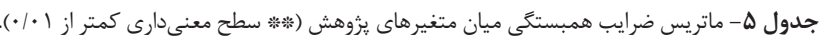

\begin{tabular}{|c|c|c|c|c|c|c|}
\hline$\Delta$ & F & $r$ & $r$ & 1 & متغير & رديف \\
\hline & & & & 1 & صدا بيزارى & 1 \\
\hline & & & 1 & $\cdot / \wedge v^{* *}$ & علايمم صدا بيزارى & r \\
\hline & & 1 &.$/ 9 F^{* *}$ & $\cdot / 9 f^{* *}$ & هيجانات ناشى از صدا بيزارى & $r$ \\
\hline & 1 & $\cdot / \mu p^{* *}$ & $\cdot / r \Lambda^{* *}$ & $\cdot / r \Delta^{* *}$ & وسواس فكرى -عملى & $p$ \\
\hline 1 & $\cdot / \Delta 0^{* *}$ & $\cdot / r 8^{* *}$ & $\cdot / r \Delta^{* *}$ & $\cdot / 4 q^{* *}$ & افسردكى & $\Delta$ \\
\hline$\cdot / \Delta \Delta^{* *}$ & $\cdot / \& V^{* *}$ & $\cdot / \& v^{* *}$ & $\cdot / F r^{* *}$ & $\cdot / \Delta \theta^{* *}$ & اضطراب & 9 \\
\hline
\end{tabular}

${ }^{34} \mathrm{Zhou}, \mathrm{Wu}$, and Storch 


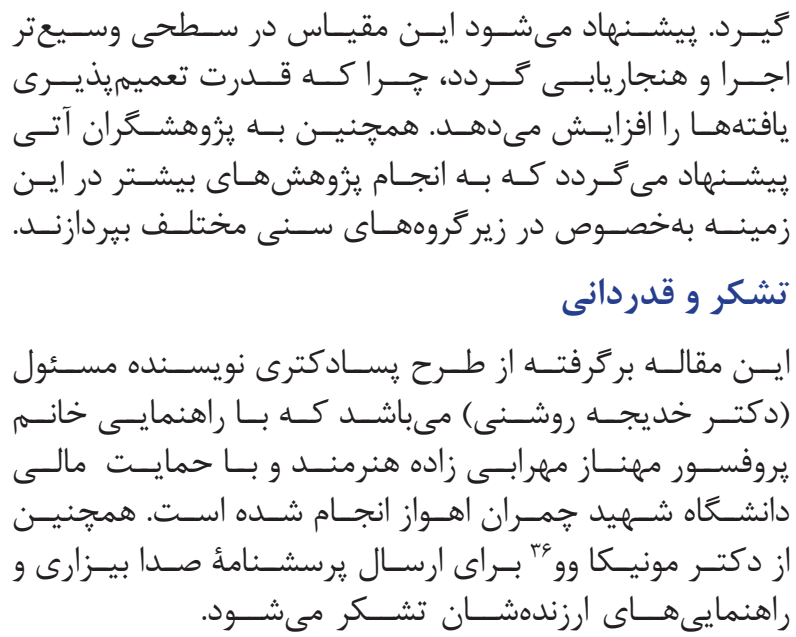

1. Jastreboff PJ, Jastreboff MM. Treatments for decreased sound tolerance (Hyperacusis and Misophonia). Seminars in Hearing. 2014; 35(2): 105-20.

2. Rouw R, Erfanian M. A large-scale study of misophonia. J Clin Psychol. 2018; 74(3): 453-79.

3. Dozier TH. Understanding and overcoming misophonia: a conditioned aversive reflex disorder. Misophonia Treatment Institute, Livermore, CA, USA. 2015; p.11-22.

4. Bruxner G. Mastication rage: a review of misophoniaAn under- recognised symptom of psychiatric relevance? Australas Psychiatry. 2016; 24(2): 195-7.

5. Jastreboff PJ, Hazell, JWP. Tinnitus retraining therapy: Implementing the neurophysiological model. Cambridge University Press. 2008.

6. Schwartz P, Leyendecker J, Conlon M. Hyperacusis and misophonia: the lesser-known siblings of tinnitus. Minn Med. 2011; 94(11): 42-3.

7. Taylor S. Misophonia: a new mental disorder? Med Hypotheses. 2017; 103: 109-17.

8. Schröder A, Vulink N, Denys D. Misophonia: diagnostic criteria for a new psychiatric disorder. PLoS One. 2013; 8(1): e54706. doi: 10.1371/journal. pone. 0054706 .

9. Wu MS, Lewin AB, Murphy TK, Storch EA. Misophonia: incidence, phenomenology, and clinical correlates in an undergraduate student sample. J Clin Psychol. 2014; 70(10): 994-1007.

10. Mousavi M, Parvin, S, Farid F, Bahrainian A,

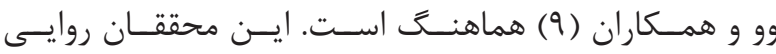

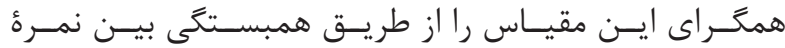

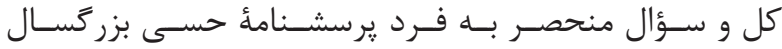

$$
\begin{aligned}
& \text { هـ (ASQ) }
\end{aligned}
$$

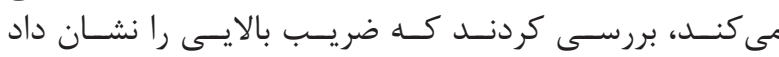

$$
\begin{aligned}
& \text { (r= } 10 \cdot, P<\cdot 1 \cdot \cdot 1) \\
& \text { در مجمـوع، نتايـج به دسـت آمــده از يزوهــش حاضر نشـان داد }
\end{aligned}
$$

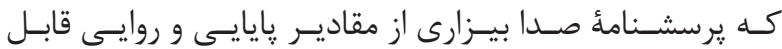

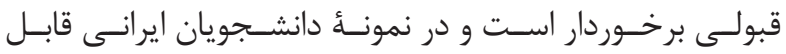

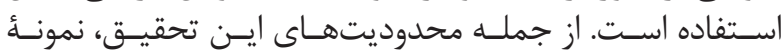

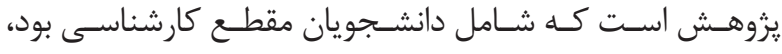

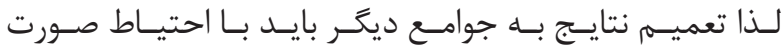

$$
\begin{aligned}
& \text { منابع }
\end{aligned}
$$

Asghanejad Farid A. Evaluation of emotional intelligence training efficiency aimed at improving the quality of life, reducing symptoms of anxiety and depression among the elderly in Tehran nursing home. Journal of Fundamentals of Mental Health. 2016; 24(18): 520-6.

11. Kaviani H, Mousavi AS. Psychometric properties of the Persian version of Beck Anxiety Inventory (BAI). TUMJ. 2008; 66(2): 136-40.

12. Beck AT, Steer RA, Ball R, Ranieri W. Comparison of beck depression inventories -ia and -ii in psychiatric outpatients. J Pers Assess. 1996; 67(3): 588-97.

13. Stefan-Dabson K, Mohammadkhani P, MassahChoulabi O. Psychometrics characteristic of beck depression inventory-ii in patients with major depressive disorder. Journal of Rehabilitation. 2007; 8: 82-0.

14. Rajabi G, Attari Y, Haghighi J. Factorial analysis of beck depression inquiry on male students of ahvaz shahid chamran university. Journal Educational Sciences and Psychology. 2001; 8(3-4): 49-66.

15. Goodman WK, Price LH, Rasmussen SA, Mazure C, Delgado P, Heniger GR, et al. The yale-brown obsessive compulsive scale. II. Validity. Arch Gen Psychiatry. 1989; 46(11): 1012-6.

16. Deacon BJ, Abramowitz JS. The yale-brown obsessive compulsive scale: factor analysis, construct validity, and suggestions for refinement. J Anxiety Disord. 2005; 19(5): 573-85.

17. Eisen JL, Phillips KA, Baer L, Beer DA, Atala KD, Rasmussen SA. The brown assessment of beliefs scale: reliability and validity. Am J Psychiatry. 1998; 155(1): 102-8.

${ }^{35}$ Adult sensory questionnaire

${ }^{36}$ Monica Wu 
18. Amani M, Abolghasemi A, Ahadi B, Narimani M. The effectiveness of inference-based therapy on reduction of obsession and compulsion symptoms. Research in Clinical Psychology and Counseling, 2013; 3(1): $55-68$.

19. Colquit JA. On the dimensionality of organizational justice: A construct validation of a measure. J Appl Psychol. 2001; 86(3): 386-400.

20. Zhoua X, Wu MS, Storch EA. Misophonia symptoms among Chinese university students: Incidence, associated impairment, and clinical correlates. Journal of ObsessiveCompulsive and Related Disorders. 2017; 14: 7-12. 